\title{
An Overview Research on Wireless Communication Network
}

\author{
Mohaiminul Islam, Shangzhu Jin \\ School of Intelligent Technology and Engineering, Chongqing University of Science and Technology, Chongqing, China
}

Email address:

mohaiminul100@hotmail.com (M. Islam), szjin@cqust.edu.cn (Shangzhu Jin)

\section{To cite this article:}

Mohaiminul Islam, Shangzhu Jin. An Overview Research on Wireless Communication Network. Advances in Wireless Communications and Networks. Vol. 5, No. 1, 2019, pp. 19-28. doi: 10.11648/j.awcn.20190501.13

Received: July 30, 2019; Accepted: August 27, 2019; Published: September 11, 2019

\begin{abstract}
Communication Systems can be Wired or Wireless and the medium used for communication can be Guided or Unguided. In Wired Communication, the medium is a physical path like Co-axial Cables, Twisted Pair Cables and Optical Fiber Links etc. which guides the signal to propagate from one point to other. Such type of medium is called Guided Medium. The term wireless refers to the communication or transmission of information over a distance without requiring wires, cables or any other electrical conductors. Wireless communication is one of the important mediums of transmission of data or information to other devices. The Communication is set and the information is transmitted through the air, without requiring any cables, by using electromagnetic waves like radio frequencies, infrared, satellite, etc., in a wireless communication technology network. At the end of the 19th century, the first wireless communication systems were introduced and the technology has significantly been developed over the intervening and subsequent years. Today, the term wireless refers to a variety of devices and technologies ranging from smart phones to laptops, tabs, computers, printers, Bluetooth, etc. On the other hand, Wireless Communication doesn't require any physical medium but propagates the signal through space. Since, space only allows for signal transmission without any guidance, the medium used in Wireless Communication is called Unguided Medium. In the present days, wireless communication system has become an essential part of various types of wireless communication devices, that permits user to communicate even from remote operated areas. There are many devices used for wireless communication like mobiles. Cordless telephones, GPS, Wi-Fi, satellite television and wireless computer parts. Current wireless phones include 3 and 4G networks, Bluetooth and Wi-Fi technologies. This paper is focused on elements of Wireless Communication system, Types of Wireless Communication, Advantage \& Disadvantage of it, Smart city, wireless network security.
\end{abstract}

Keywords: Wireless Communication, Bluetooth, WALNs, Infrared Communication, Transmission, Network

\section{Introduction}

Wireless communication plays a significant role in day to day life. Besides communication, wireless technology has become an integral part of our daily activities. The transmission of data or information from one place to another witlessly is referred as wireless communication. This provides an exchange of data without any conductor through RF and radio signals. The information is transmitted across the devices over some meters to hundreds of kilometers through well-defined channels. The term wireless refers to communication without wires. In order to transmit information (voice or data) using wireless communication we need antenna. The antenna is the device which couples RF energy from one medium (i.e. wave guide, transmission line etc.) to the other medium (i.e. air). We require two systems viz. transmitter and receive to complete end to end wireless link. Wireless communication uses electromagnetic waves as medium for carrying the information through the channel between transmitter and receiver [1].

The term wireless refers to communication without wires. In order to transmit information (voice or data) using wireless communication we need antenna. The antenna is the device which couples RF energy from one medium (i.e. waveguide, transmission line etc.) to the other medium (i.e. air). We require two systems viz. transmitter and receive to complete end to end wireless link. Wireless communication uses electromagnetic waves as medium for carrying the information through the channel between transmitter and receiver. The wireless communication revolution is bringing 
fundamental changes to data networking, telecommunication, and is making integrated networks a reality. By freeing the user from the cord, personal communications networks, wireless LAN's, mobile radio networks and cellular systems, harbor the promise of fully distributed mobile computing and communications, anytime, anywhere. Focusing on the networking and user aspects of the field, Wireless Networks provides a global forum for archival value contributions documenting these fast growing areas of interest. The journal publishes refereed articles dealing with research, experience and management issues of wireless networks. Its aim is to allow the reader to benefit from experience, problems and solutions described. The primary and important benefit of wireless communication is mobility. Apart from mobility, wireless communication also offers flexibility and ease of use, which makes it increasingly popular day - by - day. Wireless Communication like mobile telephony can be made anywhere and anytime with a considerably high throughput performance $[2,3]$.

Another important point is infrastructure. The setup and installation of infrastructure for wired communication systems is an expensive and time consuming job. The infrastructure for wireless communication can be installed easily and low cost.

In emergency situations and remote locations, where the setup of wired communication is difficult, wireless communication is a viable option.

\section{Wireless Communication}

Heinrich Herz discovered and first produced radio waves in 1888 and by 1894 the modern way to send a message over telegraph wires was first conducted. Marconi sent and received signals up to two miles using radio waves. Marconi became known as the father of radio. By 1899, Marconi sent a signal nine miles across the Bristol Channel and 31 miles across the English Channel to France. In 1901 he was able to transmit across the Atlantic Ocean.

During World War II, the United States Army first used radio signals for data transmission. This inspired a group of researchers in 1971 at the University of Hawaii to create the first packet based radio communications network called ALOHNET. ALOHNET was the very first wireless local area network (WLAN). This first WLAN consisted of 7 computers that communicated in a bi-directional star topology. The first generation of WLAN technology used an unlicensed band (902-928 MHz ISM), which later became crowded with interference from small appliances and industrial machinery. A spread spectrum was used to minimize this interference, which operated at 500 kilobits per second. The second generation of WLAN technology was four times faster and operating at $2 \mathrm{Mbps}$ per second. Third generation WLAN technology operates on the same band as the second generation and we currently use it today [4].

In 1990, the IEEE 802 Executive Committee established the 802.11 Working Group to create a wireless local area network (WLAN) standard. The standard specified an operating frequency in the $2.4 \mathrm{GHz}$ ISM band. In 1997 the group approved IEEE 802.11 as the world's first WLAN standard with data rates of 1 and 2 Mbps.

\subsection{WPANS: Wireless Personal Area Networks}

A wireless personal area network (WPAN) is a personal, short distance area wireless network for interconnecting devices centered around an individual person's workspace.WPANs address wireless networking and mobile computing devices such as Personal Computers, Personal Digital Assistants, peripherals, cell phones, pagers and consumer electronics. WPANs are also called short wireless distance networks. The two current technologies for wireless personal area networks are Infra Red (IR) and Bluetooth (IEEE 802.15). These will allow the connectivity of personal devices within an area of about 30 feet. However, IR requires a direct line of site and the range is less.

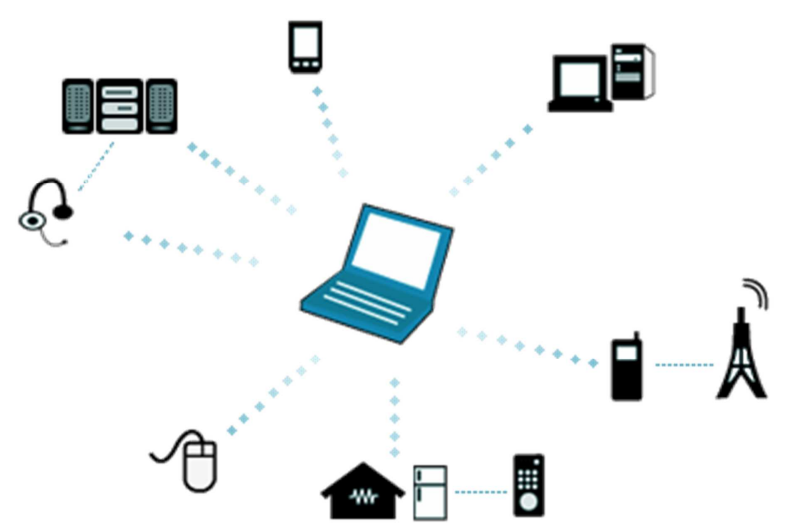

Figure 1. WPANS.

\subsection{WLANS: Wireless Local Area Networks}

WLANS allow users in a local area, such as a university campus or library, to form a network or gain access to the internet. A temporary network can be formed by a small number of users without the need of an access point; given that they do not need access to network resources. It implements a flexible data communication system frequently augmenting rather than replacing a wired LAN within a building or campus. WLANs use radio frequency to transmit and receive data over the air, minimizing the need for wired connections. A wireless local area network (WLAN) is a wireless distribution method for two or more devices that use high-frequency radio waves and often include an access point to the Internet. A WLAN allows users to move around the coverage area, often a home or small office, while maintaining a network connection [5].

\subsection{WMANS: Wireless Metropolitan Area Networks}

This technology allows the connection of multiple networks in a metropolitan area such as different buildings in a city, which can be an alternative or backup to laying copper or fiber cabling. A Wireless Metropolitan Area Network (WMAN) is also known as a Wireless Local Loop (WLL).WMANs are based on the IEEE 802.16 standard. Wireless local loop can 
reach effective transfer speeds of 1 to $10 \mathrm{Mbps}$ within a range of 4 to 10 kilometres, which makes it useful mainly for telecommunications companies.

\subsection{WWANS: Wireless Wide Area Networks}

These types of networks can be maintained over large areas, such as cities or countries, via multiple satellite systems or antenna sites looked after by an Internet Service Provider (ure
4). Thes types of systems are referred to as $2 \mathrm{G}$ (2nd Generation) systems. The three families of WWAN technologies are GSM/UMTS, CDMA One/CDMA 2000 and WiMAX. In the United States, service providers include AT \& T, Clearwire, Sprint and Verizon. Wireless WAN services are expected to become increasingly available as $4 \mathrm{G}$ technologies mature.

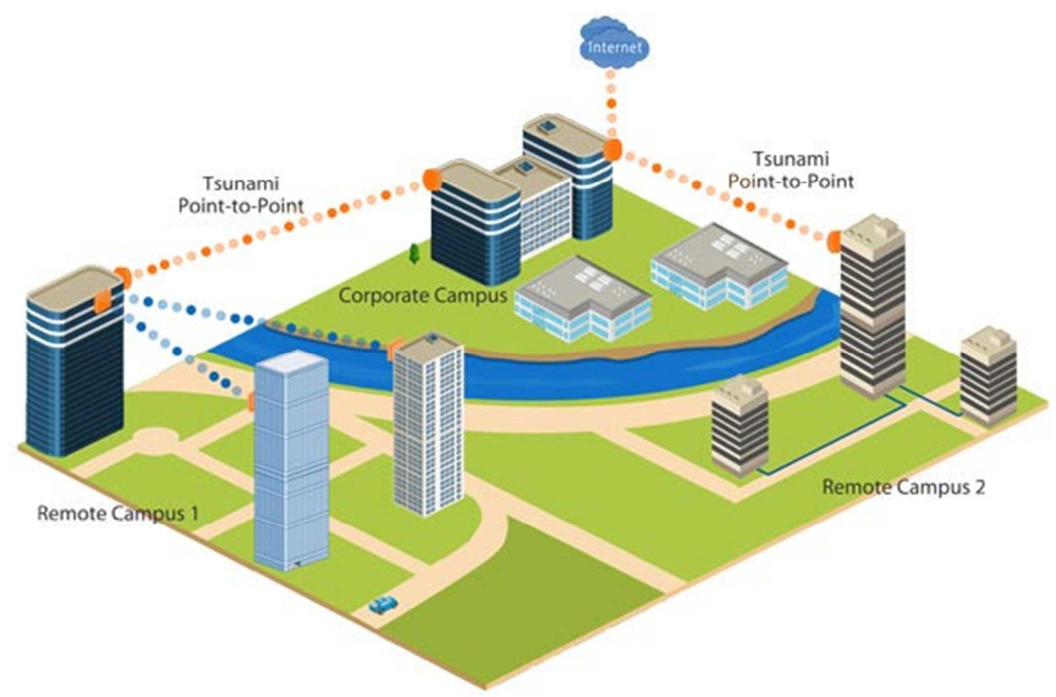

Figure 2. WMANS.

\subsection{Cellular Networks}

A cellular network or mobile network is a wireless network distributed over land areas called cells, each served by at least one fixed-location transceiver, known as a cell site or base station. In a cellular network, each cell uses a different set of frequencies from neighboring cells, to avoid interference and provide guaranteed bandwidth within each cell. When joined together these cells provide radio coverage over a wide geographic area. This enables a large number of porfigur transceivers (e.g., mobile phones, pagers, etc.) to communicate with each other and with fixed transceivers and telephones anywhere in the network, via base stations, even if some of the transceivers are moving through more than one cell during transmission [6].

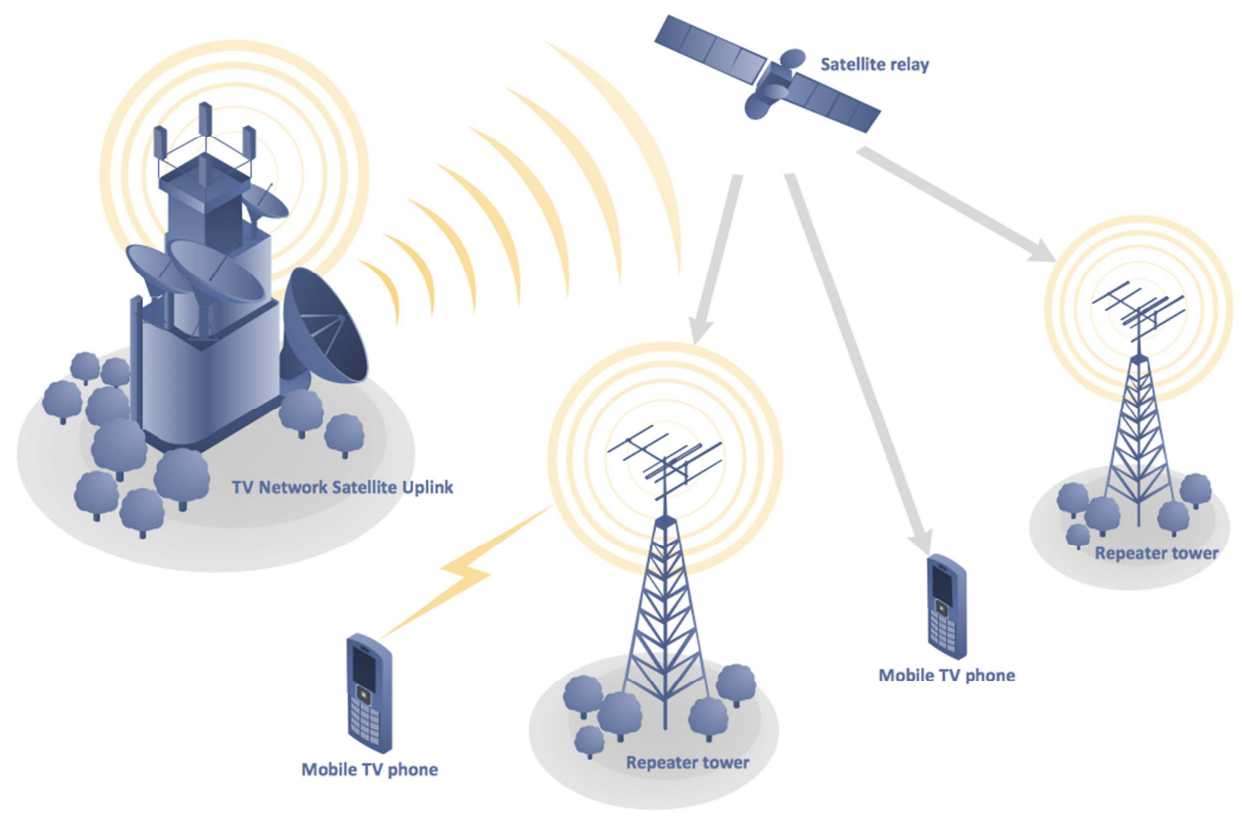

Figure 3. Wireless Cellular Network. 


\subsection{VPN: Virtual Private Network}

A virtual private network (VPN) extends a private network across a public network, such as the Internet. It enables a computer or network-enabled device to send and receive data across shared or public networks as if it were directly connected to the private network, while benefiting from the functionality, security and management policies of the private network. A VPN is created by establishing a virtual point-to-point connection through the use of dedicated connections, virtual tunneling protocols, or traffic encryptions. Major implementations of VPNs include Open VPN and IPsec.

\section{Basic Elements of a Wireless Communication System}

A typical Wireless Communication System can be divided into three elements: the Transmitter, the Channel and the Receiver. The following image shows the block diagram of wireless communication system.

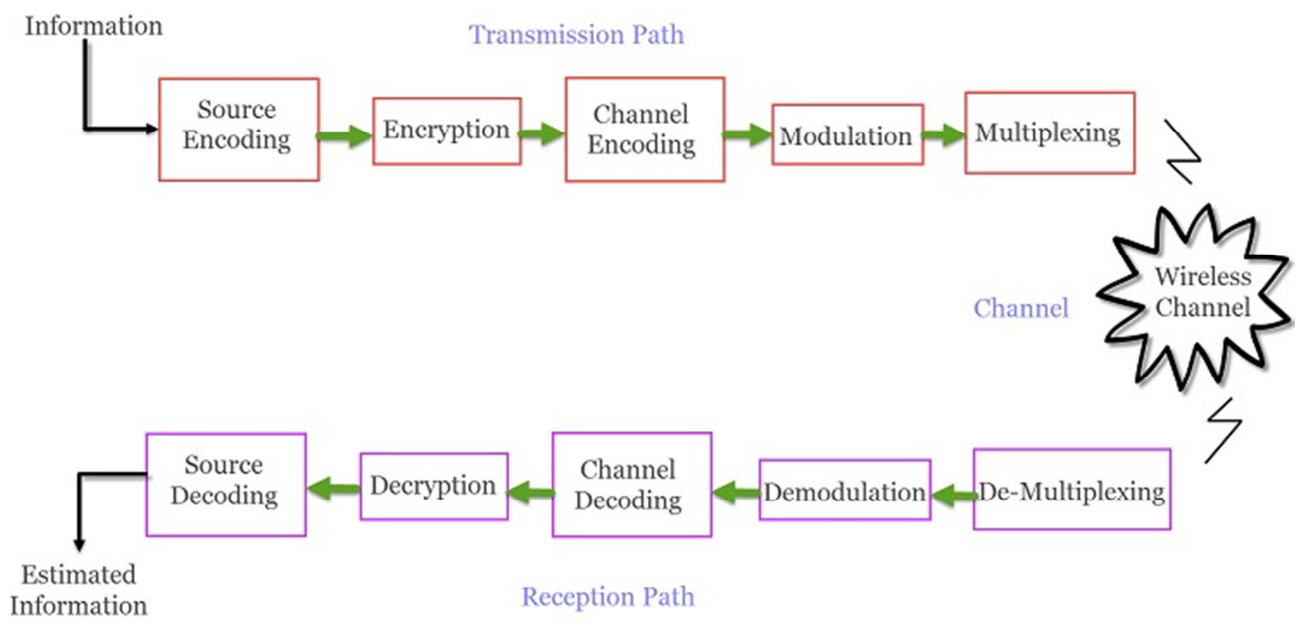

Figure 4. Block diagram of WCS.

\subsection{The Transmission Path}

A typical transmission path of a Wireless Communication System consists of Encoder, Encryption, Modulation and Multiplexing. The signal from the source is passed through a Source Encoder, which converts the signal in to a suitable form for applying signal processing techniques.

The redundant information from signal is removed in this process in order to maximize the utilization of resources. This signal is then encrypted using an Encryption Standard so that the signal and the information is secured and doesn't allow any unauthorized access.

Channel Encoding is a technique that is applied to the signal to reduce the impairments like noise, interference, etc. During this process, a small amount of redundancy is introduced to the signal so that it becomes robust against noise. Then the signal is modulated using a suitable Modulation Technique (like PSK, FSK and QPSK etc.), so that the signal can be easily transmitted using antenna [7].

The modulated signal is then multiplexed with other signals using different Multiplexing Techniques like Time Division Multiplexing (TDM) or Frequency Division Multiplexing (FDM) to share the valuable bandwidth.

\subsection{The Channel}

The channel in Wireless Communication indicates the medium of transmission of the signal i.e. open space. A wireless channel is unpredictable and also highly variable and random in nature. A channel maybe subject to interference, distortion, noise, scattering etc. and the result is that the received signal may be filled with errors.

\subsection{The Reception Path}

The job of the Receiver is to collect the signal from the channel and reproduce it as the source signal. The reception path of a Wireless Communication System comprises of Demultiplexing, Demodulation, Channel Decoding, Decryption and Source Decoding. From the components of the reception path it is clear that the task of the receiver is just the inverse to that of transmitter.

The signal from the channel is received by the Demultiplexer and is separated from other signals. The individual signals are demodulated using appropriate Demodulation Techniques and the original message signal is recovered. The redundant bits from the message are removed using the Channel Decoder.

Since the message is encrypted, Decryption of the signal removes the security and turns it into simple sequence of bits. Finally, this signal is given to the Source Decoder to get back the original transmitted message or signal $[8,9]$.

\section{Types of Wireless Communication}

The different types of wireless communication mainly include IR wireless communication, satellite communication, 
broadcast radio, Microwave radio, Bluetooth, Zigbee etc.

\subsection{Satellite Communication}

Satellite communication is one type of self contained wireless communication technology; it is widely spread all over the world to allow users to stay connected almost anywhere on the earth. When the signal (a beam of modulated microwave) is sent near the satellite then, satellite amplifies the signal and sent it back to the antenna receiver which is located on the surface of the earth. Satellite communication contains two main components like the space segment and the ground segment.The ground segment consists of fixed or mobile transmission, reception and ancillary equipment and the space segment, which mainly is the satellite itself.

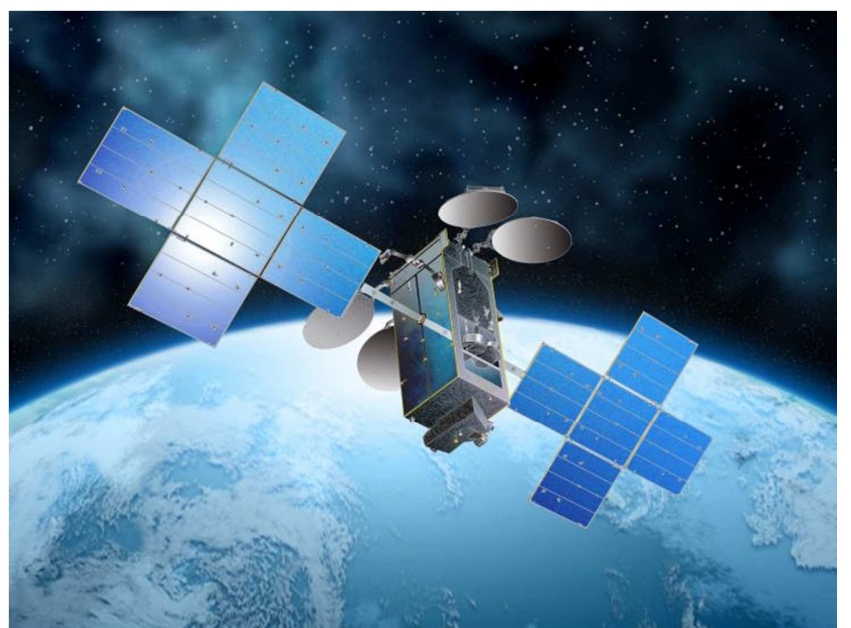

Figure 5. Satellite Communication.

\subsection{Infrared Communication}

Infrared communicates information in a device or systems through IR radiation. IR is electromagnetic energy at a wavelength that is longer than that of red light. It is used for security control, TV remote control and short range communications. In the electromagnetic spectrum, IR radiation lies between microwaves and visible light. So, they can be used as a source of communication.

For a successful infrared communication, a photo LED transmitter and a photo diode receptor are required. The LED transmitter transmits the IR signal in the form of non visible light that is captured and saved by the photoreceptor. So the information between the source and the target is transferred in this way. The source and destination can be mobile phones, TVs, security systems, laptops etc supports wireless communication [10].

\subsection{Broadcast Radio}

The first wireless communication technology is the open radio communication to seek out widespread use, and it still serves a purpose nowadays. Handy multichannel radios permit a user to speak over short distances, whereas citizen's band and maritime radios offer communication services for sailors. Ham radio enthusiasts share data and function emergency communication aids throughout disasters with their powerful broadcasting gear, and can even communicate digital information over the radio frequency spectrum.

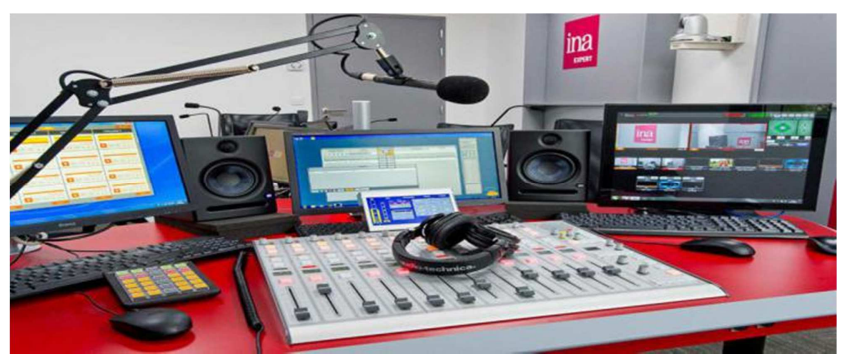

Figure 6. Broadcast Radio Studio.

Mostly an audio broadcasting service, radio broadcasts sound through the air as radio waves. Radio uses a transmitter which is used to transmit the data in the form of radio waves to a receiving antenna (Different Types of Antennas). To broadcast common programming, stations are associated with the radio N/W's. The broadcast happens either in simulcast or syndication or both. Radio broadcasting may be done via cable FM, the net and satellites. A broadcast sends information over long distances at up to two megabits/Sec (AM/FM Radio) [11].

Radio waves are electromagnetic signals, that are transmitted by an antenna.These waves have completely different frequency segments, and you will be ready to obtain an audio signal by changing into a frequency segment.

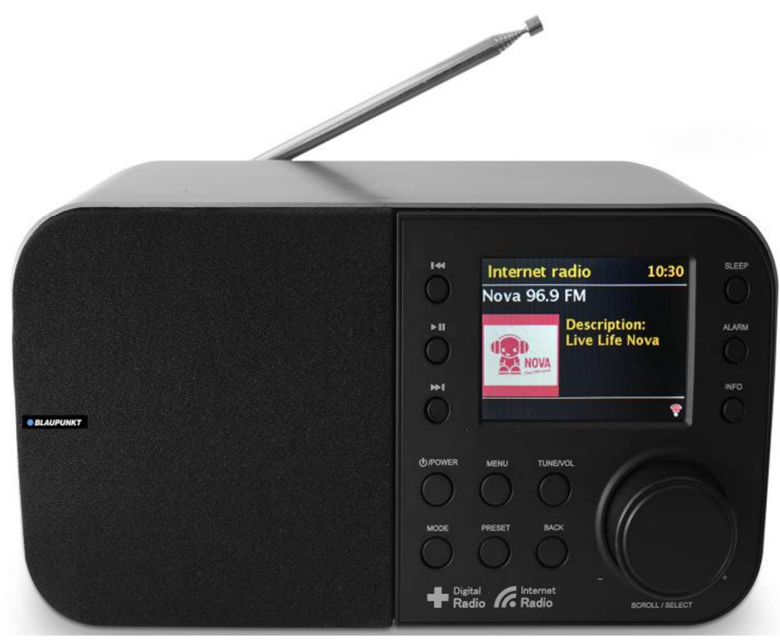

Figure 7. Radio

For example, you can take a radio station. When the RJ says you are listening to 92.7 BIG FM, what he really means is that signals are being broadcast at a frequency of 92.7 megahertz, that successively means the transmitter at the station is periodic at a frequency of 92.700,000 Cycles/second.

When you would like to listen to 92.7 BIG FM, all you have to do is tune the radio to just accept that specific frequency and you will receive perfect audio reception.

\subsection{Microwave Communication}

Microwave wireless communication is an effective type of 
communication, mainly this transmission uses radio waves, and the wavelengths of radio waves are measured in centimeters. In this communication, the data or information can be transfers using two methods. One is satellite method and another one is terrestrial method.

Wherein satellite method, the data can be transmitted though a satellite, that orbit 22,300 miles above the earth. Stations on the earth send and receive data signals from the satellite with a frequency ranging from $11 \mathrm{GHz}-14 \mathrm{GHz}$ and with a transmission speed of $1 \mathrm{Mbps}$ to $10 \mathrm{Mbps}$. In terrestrial method, in which two microwave towers with a clear line of sight between them are used, ensuring no obstacles to disrupt the line of sight. So it is used often for the purpose of privacy. The frequency range of the terrestrial system is typically $4 \mathrm{GHz}-6 \mathrm{GHz}$ and with a transmission speed is usually $1 \mathrm{Mbps}$ to $10 \mathrm{Mbps}$.

The main disadvantage of microwave signals is, they can be affected by bad weather, especially rain.

\section{5. $\mathrm{Wi}-\mathrm{Fi}$}

Wi-Fi is a low power wireless communication, that is used by various electronic devices like smart phones, laptops, etc.In this setup, a router works as a communication hub wirelessly. These networks allow users to connect only within close proximity to a router. WiFi is very common in networking applications which affords portability wirelessly. These networks need to be protected with passwords for the purpose of security, otherwise it will access by others.

\subsection{Mobile Communication Systems}

The advancement of mobile networks is enumerated by generations. Many users communicate across a single frequency band through mobile phones. Cellular and cordless phones are two examples of devices which make use of wireless signals. Typically, cell phones have a larger range of networks to provide a coverage. But, Cordless phones have a limited range. Similar to GPS devices, some phones make use of signals from satellites to communicate [12].

\subsection{Bluetooth Technology}

The main function of the Bluetooth technology is that permits you to connect a various electronic devices wirelessly to a system for the transferring of data.Cell phones are connected to hands free earphones, mouse, wireless keyboard. By using Bluetooth device the information from one device to another device, this technology has various functions and it is used commonly in the wireless communication market.

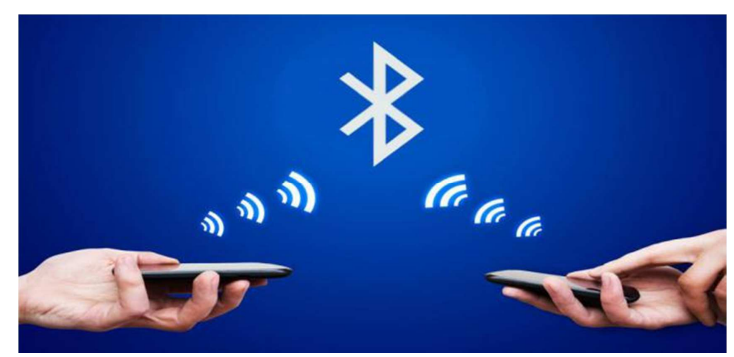

Figure 8. Bluetooth.

\section{Advantages of Wireless Communication}

There are numerous advantages of Wireless Communication Technology, Wireless Networking and Wireless Systems over Wired Communication like Cost, Mobility, Ease of Installation, and Reliability etc.

\subsection{Cost}

The cost of installing wires, cables and other infrastructure is eliminated in wireless communication and hence lowering the overall cost of the system compared to wired communication system. Installing wired network in building, digging up the Earth to lay the cables and running those wires across the streets is extremely difficult, costly and time consuming job.

In historical buildings, drilling holes for cables is not a best idea as it destroys the integrity and importance of the building. Also, in older buildings with no dedicated lines for communication, wireless communication like Wi-Fi or Wireless LAN is the only option.

\subsection{Mobility}

As mentioned earlier, mobility is the main advantage of wireless communication system. It offers the freedom to move around while still connected to network [13].

\subsection{Ease of Installation}

The setup and installation of wireless communication network's equipment and infrastructure is very easy as we need not worry about the hassle of cables. Also, the time required to setup a wireless system like a Wi-Fi network for example, is very less when compared to setting up a full cabled network [14].

\subsection{Reliability}

Since there are no cables and wires involved in wireless communication, there is no chance of communication failure due to damage of these cables which may be caused by environmental conditions, cable splice and natural diminution of metallic conductors.

\subsection{Disaster Recovery}

In case of accidents due to fire, floods or other disasters, the loss of communication infrastructure in wireless communication system can be minimal.

\section{Disadvantages of Wireless Communication}

Even though wireless communication has a number of advantages over wired communication, there are a few disadvantages as well. The most concerning disadvantages are Interference, Security and Health. 
Interference:

Wireless Communication systems use open space as the medium for transmitting signals. As a result, there is a huge chance that radio signals from one wireless communication system or network might interfere with other signals.

The best example is Bluetooth and Wi-Fi (WLAN). Both these technologies use the $2.4 \mathrm{GHz}$ frequency for communication and when both of these devices are active at the same time, there is a chance of interference.

Security:

One of the main concerns of wireless communication is Security of the data. Since the signals are transmitted in open space, it is possible that an intruder can intercept the signals and copy sensitive information.

Health Concerns:

Continuous exposure to any type of radiation can be hazardous. Even though the levels of RF energy that can cause the damage are not accurately established, it is advised to avoid $\mathrm{RF}$ radiation to the maximum.

\section{Wireless Communication Network Security}

Wireless communication is the transfer of information between two or more points that are not connected by an electrical conductor. A wireless network enables people to communicate and access applications and information without wires.Network security consists of the provisions and policies adopted by a network administrator to prevent and monitor unauthorized access, misuse, modification, or denial of a computer network and network-accessible resources. Cryptography is a method of storing and transmitting data in a particular form so that only those for whom it is intended can read and process it.The main aim of this article is to communicate the concept of Wireless communication, Network security and Cryptography. Wireless network security primarily protects a wireless network from unauthorized and malicious access attempts. Typically, wireless network security is delivered through wireless devices (usually a wireless router/switch) that encrypts and secures all wireless communication by default. Even if the wireless network security is compromised, the hacker is not able to view the content of the traffic/packet in transit. Moreover, wireless intrusion detection and prevention systems also enable protection of a wireless network by alerting the wireless network administrator in case of a security breach. Some of the common algorithms and standards to ensure wireless network security are Wired Equivalent Policy (WEP) and Wireless Protected Access (WPA) [15].

\section{Smart City and Wireless Communication Network}

The term Smart Cities has taken on a lot of traction lately and now ranks as a full-fledged buzzword. But, as is the case with most buzzwords, its meaning is a bit vague and practical applications and specific benefits often unclear to most people. Fluid-mesh, however, has had the privilege of being involved with many projects for cities in countries around the world, and I learned, by implementing real-life projects, what a Smart City is and what it means in practice. Practical implementations of the Smart City concept often involve technology and automation. Among the goals of a Smart City is providing security and protection for the people living in the Smart City. The work Fluid-mesh has done with and its expertise in security-related projects with law enforcement agencies has given me clear insight into what a Smart City actually is. Over the past decade, many cities and towns around the world have invested a significant amount of capital to deploy urban video-surveillance systems. We've all seen these systems in action in the media during major acts of terror - and they've often led to the identification and arrest of the criminals behind these attacks. Having many video feeds that can be used to protect people and prosecute criminals is a critical component of every Smart City.

Also relative to video streaming in a Smart City is situational awareness: government agencies and organizations in charge of safety, security and emergency management use video streams in real time in order to react to disasters and save lives. Urban security cameras are the most important element of an emergency detection system, but local government agencies also use other types of sensors in conjunction with video feeds. These can include gun-shot detection sensors and chemical, biological and nuclear sensors, which are commonly used in large cities that might be a target for an attack. Beyond security and safety, other major components of a Smart City that I've had direct experience in with Fluid-mesh are smart and sustainable mobility. Ideally, a Smart City is not a place where people can only use their personal vehicles and get stuck in traffic for hours. People should be able to travel on mass transit systems that are energy-efficient and faster than personal vehicles. Fluid-mesh has been involved in projects that require automating traffic signals based on the location of mass transit vehicles and light rail systems. These systems are a reality today, although many people don't realize it [16]. Typically, when a modern tram or surface metro approaches an intersection, the tram or train is able to immediately turn the traffic signal to green and proceed without stopping at the intersection. Similar automation can be implemented in the future for cars and traffic lights to control the timing of a traffic light based on traffic flow. Another interesting practical application involves parking. According to Cisco, up to $40 \%$ of the traffic in city centers is generated by people driving around looking for a parking spot. I was surprised at first by this figure, but it relates very well to my experience in large cities in the U.S. and Europe. People often choose not to drive into a city for fear they won't be able to find a place to park their car when they get there. Being able to use technology to quickly pinpoint where a parking spot is available would be a great help for people, and reduce traffic! This is another example of a real-life Smart City application that is just a few years away [17]. 
As a broadband wireless vendor, Fluid-mesh gets involved in many Smart City projects that's because trenching and wiring a city can be extremely expensive and using wireless transmission is typically easier in order to create the required communication network. Moreover, wireless is often the only viable way to connect the network vehicles, trams, trains and ferries, all of which are constantly moving. For example, Fluid-mesh R\&D efforts that brought our solution to connect trains and mass transit vehicles to market started five years ago because many of the cities that were already working with our products were looking for a solution to extend their network connectivity to moving vehicles. Wireless communication plays a critical role in all Smart City projects and cities very often start building a wireless network as a first step towards becoming a "Smarter City". In order to automate any process and transfer information, we're always in need of communication networks across the entire city. Back when we started Fluid-mesh in 2005, most cities were mainly looking at municipal Wi-Fi (Muni Wi-Fi) as the first step towards becoming more connected cities. The Smart City concept was still in its infancy, but the success of Wi-Fi technology catalyzed many city administrations to look at ways to build citywide Wi-Fi coverage. Unfortunately, this initial effort wasn't particularly successful because of a significant conflict of interest between, on one hand, the city offering a best-effort Wi-Fi coverage using taxpayer money, and on the other hand, many telecom operators investing their resources to enhance their cellular and $3 \mathrm{G}$ networks to provide a broadband connection to their subscribers. Moreover, ten years ago, managing and maintaining a citywide Wi-Fi network with very small cells was extremely complex and expensive.

\section{SMART CITY}

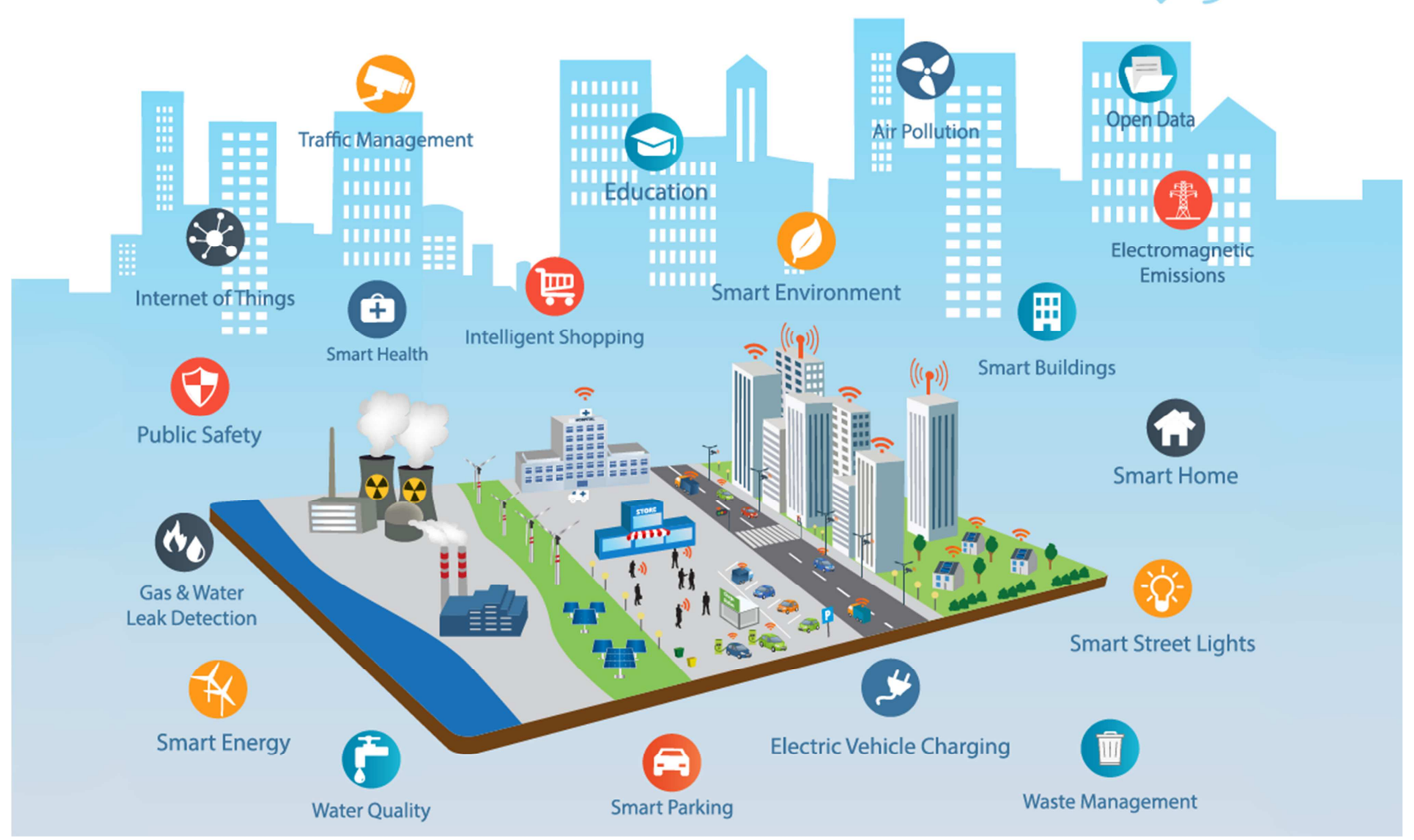

Figure 9. Future Smart City.

Today, there are still many cities that offer free Wi-Fi in areas like parks and major central areas. The focus has shifted, however, to building wireless infrastructures able to provide more than Wi-Fi services to residents and tourists. The automation piece of the Smart City concept took on traction, and providing Wi-Fi is becoming less important. This is also due to the increase in throughput available across cellular networks as a result of the deployment of LTE technology in major urban areas. The debate around free Wi-Fi provided by cities and towns is still going on, however. In addition, cellular carriers are facing challenges in keeping up with the exponential increase in throughput required by their subscribers using smart phone extensively. Therefore, large scale Wi-Fi is becoming, in certain countries, a way to offload traffic from cellular base stations (BTS) and move it on the Wi-Fi frequencies. Unlike the Muni Wi-Fi networks deployed by the cities directly in 2004-2006, telecom operators are today involved in the effort to provide connectivity to residents and tourists through widespread Wi-Fi deployments. At the same time, the focus of wireless Smart Cities in most countries is shifting away from pure Wi-Fi service; local governments are focusing more on building the wireless 
infrastructure and deploying the technology required to make people's lives safer, simpler, and more efficient. The wireless network becomes an enabler to run on top of it automation and communications systems that protect people and assets, automate processes such as garbage collection and street cleaning, and reduce traffic jams and pollution.

A wireless city is already enabled to evolve into a Smart City in the very near future. Free Wi-Fi is a very nice service and I, personally, am such a Wi-Fi lover that I prefer a hotel with a good Wi-Fi service over a cleaner one. However, I believe Wi-Fi per se does not make a city smart. Wireless technology and Wi-Fi is a critical enabler for most Smart City applications. A Smart City is one that has services running on its wireless network that allow people to get to their destination faster, walk at night with no worries, and quickly find a parking spot without wasting half an hour driving around looking for one. Smart is good!

\section{The Future of Wireless Communication Networks}

Simply put, it is impossible to take the high-frequency route at all. The frequency above $5 \mathrm{G}$ is totally useless, and the coverage is too small. Only one path can be called $5 \mathrm{G}$ if the multi-carrier system is based on LTE, at least more than three carriers. As for those who say $5 \mathrm{G}$ network system needs Gigabit level and blowing water, there is no practical application at present. (TD high-frequency mobile phones can be used under the base station, or up to 100 carriers.) Mega, but the actual long-distance, is a slag, just to catch up with wcdma. In reality, China's spectrum resources are limited, and the number of users inChinais huge. Many dense areas have emerged. The first-class signal stick and the network speed are too slow. This is the lack of bandwidth per capita, or the signal is very good, but the network can not be registered, and the bandwidth is full... So,5G, the most likely solution is multi-carrier, but multi-carrier is now defined as $4 \mathrm{G}+$, so the situation is awkward [16].In fact, the 5Gstandard has not yet been formulated, so the future $4 \mathrm{G}$ and LTE-based multi-carrier technology should also dominate our communications for several years.

The future of wireless technology is bright due to it being very cost efficient and very easy to use and the best part about it is you don't have to worry about being tangled up in wires. We have seen wireless technology in cell phones and radios. However, this technology has many other practical uses which can be seen to revolutionize the future. In straightforward and simple words wireless technology is low cost and simple to handle technology.

\subsection{The Major Wireless Technologies}

The wireless technologies are now everywhere, the development in his sector has taken it up to the standard to provide very high speed internet and mobile phone operations. Now it is possible to combine one or two wireless technologies to increase its service and efficiency. There are many types of wireless technologies like WiMax, WiFi, Bluetooth and infrared technologies. The wireless communication can take place via radio, infrared and microwave communication. The wireless applications include point to point and multi point communications. WiFi is a LAN technology which provides the facility to convert wired LAN to wireless LAN. The wireless technologies are either already incorporated into the devices or they are made compatible by installing wireless interface cards. WiMax is the next generation wireless technology aiming to overcome the deficiencies in the previous wireless systems. the reason why the wireless technologies is considered cost effective is because for employing wireless technology one does not need to change or replace existing wireless equipments. WiMax technology is also the most efficient of all the others of its type because it is aim to cover wide area networks. It can cover the range of 50 kilometers. Bluetooth technology has already replaced the need for pen drives and it is still capable of replacing wires in a LAN network [18].

\subsection{The Future Expectations from Wireless Technologies}

The word pervasive communication or ubiquitous is the future expectation of not only the enterprises but also the individuals. It is this term which gives an emerging platform to producers of wireless technology compatible products and services. Every wireless technology is expected to be everywhere always $24 / 7$ to avail the various facilities. TV broadcasting on mobile phones everywhere, very high speed broadband connectivity for users all around the world, mobile roaming at cheaper prices, real time video conferencing and calls and portable wrist sized mobile phone sets. Hence the various expectations of wireless technology are not attached entirely to the wireless broadband internet access but also to the digital gadgets. The future accounts for the complete lay down of wires from home offices and computer networks. The emergence of $4 \mathrm{G}$ is expected to give rise to wireless MAN by merging various WLANs present in one city. The cost of carrying and maintaining wireless networks is decreasing that is why it can replace wired technologies.

\subsection{The Future Challenges for Next Generation Technologies}

We are standing in the era where it is possible to have voice chats and video conferencing in even remote areas but still there are many challenges that hinder the way of wireless success. The main challenge is that how to make current network architecture compatible with next generation technologies like $4 \mathrm{G}$. This is the reason why $4 \mathrm{G}$ has not been widely adopted. The next generation technologies are modified to provide long term benefits to the technology carriers. If we look at the current Korea we would find that this region is entirely different from our world. They can make use pervasive communications throughout their country. While most of the countries in the world including USA is also looking forward to adapt to these changes in future. Hence we can say the wireless technology challenges can be overtaken 
without additional network modifications. All we need is government and individual support at all levels just like Korea [19].

\section{Conclusion}

Wireless Communication is the fastest growing and most vibrant technological areas in the communication field. Wireless Communication is a method of transmitting information from one point to other, without using any connection like wires, cables or any physical medium. Generally, in a communication system, information is transmitted from transmitter to receiver that are placed over a limited distance. With the help of Wireless Communication, the transmitter and receiver can be placed anywhere between few meters (like a T.V. Remote Control) to few thousand kilometers (Satellite Communication). We live in a World of communication and Wireless Communication, in particular is a key part of our lives. Some of the commonly used Wireless Communication Systems in our day - to - day life are: Mobile Phones, GPS Receivers, Remote Controls, Bluetooth Audio and Wi-Fi etc.. It does have concerns though as with every other new advancement that is made in today's world. The issues with security regarding access to a person's personal information or the negative impact that it may seem to have on society are a few things that are holding back the progress that wireless technology could be making. With more research and experiments conducted, the problems associated with wireless communications can be reduced and make it a more significant part of the world. Wireless technology will be very important in the near future where the need for wires connecting individual devices seems to be coming to an end.

\section{References}

[1] Pahlavan, Kaveh; Krishnamurthy, Prashant (2009). Networking Fundamentals-Wide, Local and Personal Area Communications. Wiley. ISBN 978-0-470-99290-6.

[2] Linebaugh, Kate. "Medical Devices in Hospitals go wireless." Online. wsj. The Wall Street Journal. 23 May 2010. Web. 27 Oct. 2013.

[3] Paventi, Jared. "How does a Wireless Keyboard Work." Ehow. Web. 26 Oct. 2013.

[4] Jones, George. "Future Proof. How Wireless Energy Transfer Will Kill the Power Cable." MaximumPC. 14 Sept. 2010. Web. 26 Oct. 2013.
[5] F. L. Lewis. "Wireless Sensor Networks." Smart Environments: Technologies, Protocols, and Applications, ed. D. J. Cook and S. K. Das, John Wiley, New York, 2004. Automation and robotics research institute. 26 Oct. 2013.

[6] Tech Target-Definition of Wireless - Posted by Margaret Rouse (April 2 control and traffic control systems).

[7] "Wireless headphones". Retrieved 25 May 2015.

[8] "Portable Document Format (PDF)". Ijdesign. org. Archived from the original on 2017-05-14. Retrieved 2017-05-01.

[9] Walsh, Michael (1997), Cross cultural communication problems in Aboriginal Australia, Australian National University, North Australia Research Unit, pp. 7-9, ISBN 9780731528745, retrieved 25 June 2016.

[10] Xin Li. "Complexity Theory - the Holy Grail of 21st Century". Lane Dept of CSEE, West Virginia University. Archived from the original on 2013-08-15.

[11] Wyatt, Edward (10 November 2014). "Obama Asks F. C. C. to Adopt Tough Net Neutrality Rules". New York Times. Retrieved 15 November 2014.

[12] Stallings, William (2004). Data and Computer Communications (7th intl ed.). Pearson Prentice Hall. ISBN 978-0-13-183311-1.

[13] Michael Hacker, David Burghardt, Linnea Fletcher, Anthony Gordon, William Peruzzi, Richard Prestopnik, Michael Qaíssaunee, Engineering and Technology, p. 433, Cengage Learning, 2015 ISBN 1-305-85577-9.

[14] "Inductee Details - Paul Baran". National Inventors Hall of Fame. Retrieved 6 September 2017.

[15] "Inductee Details - Donald Watts Davies". National Inventors Hall of Fame. Archived from the original on 6 September 2017 Retrieved 6 September 2017.

[16] Emil Protalinski (7 April 2012). "Anonymous hacks UK government sites over 'draconian surveillance"'. ZDNet. Retrieved 12 March 2013.

[17] Bennett, Richard (September 2009). "Designed for Change: End-to-End Arguments, Internet Innovation, and the Net Neutrality Debate" (PDF). Information Technology and Innovation Foundation. p. 11. Retrieved 11 September 2017.

[18] Boyle, D.; Yates, D.; Yeatman, E. (2013). "Urban Sensor Data Streams: London 2013". IEEE Internet Computing. 17 (6): 1. doi: 10.1109/MIC.2013.85.

[19] Winkless, Laurie (11 August 2016). Science and the City: The Mechanics Behind the Metropolis. Bloomsbury Publishing. ISBN 9781472913227. 